\section{Review}

Correspondence

Nicholas J. Talbot

N.J.Talbot@exeter.ac.uk

\title{
Fungal physiology - a future perspective
}

\author{
Richard A. Wilson ${ }^{1}$ and Nicholas J. Talbot ${ }^{2}$ \\ ${ }^{1}$ Department of Plant Pathology, University of Nebraska, Lincoln, NE 68588-0660, USA \\ ${ }^{2}$ School of Biosciences, University of Exeter, Exeter EX4 4QG, UK
}

\begin{abstract}
The study of fungal physiology is set to change dramatically in the next few years as highly scalable technologies are deployed allowing accurate measurement and identification of metabolites, proteins and transcripts within cells. The advent of next-generation DNA-sequencing technologies will also provide genome sequence information from large numbers of industrially relevant and pathogenic fungal species, and allow comparative genome analysis between strains and populations of fungi. When coupled with advances in gene functional analysis, proteinprotein interaction studies, live cell imaging and mathematical modelling, this promises a stepchange in our understanding of how fungal cells operate as integrated dynamic living systems.
\end{abstract}

\section{Introduction}

While some fungi are beneficial to humankind, for instance in the production of antibiotics, food and beverages, many interactions between human populations and fungi have serious and negative socio-economic outcomes. Crop losses from plant-pathogenic fungi can, for example, be catastrophic: blast, wilt and rust fungal diseases all significantly impact upon yield, and their control is a significant component of the costs incurred by farmers (Zeigler et al., 1994; Wilson \& Talbot, 2009; Desjardins, 2003; Staples, 2000). Contamination of food and feed with mycotoxins - particularly the carcinogen aflatoxin - also has far-reaching health implications (Calvo et al., 2002). More ominously, in recent years, fungi have emerged as direct threats to human health. The rise in the number of immunocompromised patients in hospitals, due to use of immuno-suppressive therapies and the increased prevalence of autoimmune disorders, has seen a concomitant rise in fatal fungal infections of humans (Hedayati et al., 2007), while other fungal species can cause deep-seated mycoses even in immunocompetent hosts. Therefore, the study of fungi and how they respond to their environment to cause disease will likely increase in importance in the future.

How will developments in systems biology - which aims to develop predictive models of how cellular processes operate within cells and whole organisms - facilitate such investigations? It is our view that the ability to generate and mine large-scale datasets of biological molecules and their interactions, coupled with recent advances in highthroughput gene functional analysis and cell biology, will have a profound affect on our understanding of the emergent properties of the fungal cells that condition infection-related development and pathogenesis. In this perspective, we will discuss how advances in (i) rapid genomic and transcriptomic analysis, (ii) large-scale elucidation of protein-protein interaction networks and signalling pathways, (iii) metabolic flux analysis and metabolite identification, (iv) phenotypic microarray analysis, and (iv) the characterization of mutants generated by high-throughput gene deletion strategies, will advance the field of fungal physiology in future.

\section{The application of massively parallel sequencing technologies to fungal physiology}

Growth and development of fungi, including processes required for virulence, are regulated by signalling pathways that are often conserved between species (Harris et al., 2009; Rispail et al., 2009). The comparison of annotated genomes from many related species can highlight common core components of these signalling cascades, while global analyses of changes in gene expression under different environmental conditions and developmental stages can be used to identify downstream targets. However, comparative genomic studies of fungi are currently somewhat limited, due to the relative paucity of well-annotated fungal genomes and the large proportion of genes in fungi with no known function (Cornell et al., 2007; Soanes et al., 2007). Benefiting from advances in next-generation sequencing, this situation is likely to change with the routine use of affordable, massively parallel sequencing platforms such as the 454 from Roche, the Applied Biosystems SOLiD and the Illumina GA2. These instruments were designed to sequence genomes very rapidly, at a fraction of the cost associated with traditional Sanger sequencing methods. The 454 technology produces long and accurate reads of up to $500 \mathrm{bp}$, enabling rapid de novo assembly of unknown fungal genomes, while Illumina sequencing produces shorter reads (up to $70 \mathrm{bp}$ ), that normally require comparison to a reference genome to provide high-quality assemblies. The use of paired-end reads on the Illumina and new chemistries to allow read length to be extended 
beyond $100 \mathrm{bp}$ does, however, bring the realistic prospect of de novo sequencing of fungal genomes on the Illumina platform. Currently, a combination of $454 / \mathrm{SOLiD}$ and Illumina sequencing approaches is providing a powerful means of generating rapid and affordable fungal genome sequences, and we can expect the rate of publication of sequenced fungal genomes, of both new species and related isolates, to increase dramatically in the next year. Even more rapid DNA sequencing technologies are also promised by a number of companies (for example, Pacific Biosciences and their single-molecule real-time detection technology), promising ever faster and cheaper means of acquiring fungal genomic information.

We envisage that these new sequencing approaches will be key to identifying the core inventory of genes in both freeliving and symbiotic fungal species, including pathogenicity-associated gene functions in human and plant fungal pathogens and the diverse set of genes encoding enzymes involved in secondary metabolic pathways (as demonstrated by Yadav et al., 2009), all of which are likely to be highly diverse in fungi. Moreover, the identification of effector-encoding genes is likely to be a priority in the study of pathogenic fungal species. In bacterial pathogens, the identification of the type III secretion system was fundamental to the discovery of effector proteins in both human and plant pathogens (Galán \& Wolf-Watz, 2006). Bacterial type III effectors are delivered into host cells, where they suppress innate immunity responses, perturb cell signalling and condition intracellular colonization of tissues by some human-pathogenic bacteria. They are often characterized by mimicry of eukaryotic cell signalling proteins, including guanine nucleotide exchange factors, and GTPase-activating proteins. How pathogenic fungi deliver proteins into the cytoplasm of their hosts is not known and it is also unclear whether they also utilize specialized secretory pathways during pathogenic interactions. Comparative genomics and functional studies promise to allow identification of novel mechanisms of protein delivery, if they exist in fungi, and also to determine whether fungal pathogens deploy large numbers of effector proteins during infections. Interestingly, the oomycetes, which cause important plant diseases and are filamentous osmotrophic eukaryotes closely resembling fungi, secrete effector proteins into host plant cells and utilize a mechanism that appears to be conserved in the malaria pathogen of humans (Whisson et al., 2007).

Comparing the genomes of clinical or plant-pathogenic strains of a particular fungal species using next-generation sequencing technology can also allow the use of an evolutionary approach to study gene function. By identifying the most polymorphic genes within a genome, for instance, focusing particularly on those encoding secreted proteins that are expressed during pathogenesis, genes can be identified that are evolving rapidly in order to escape detection by host defences. By analysing the relative frequency of non-synonymous and synonymous substitutions in genes, the most highly polymorphic genes can be identified. These genes are ideal candidates for functional analysis, as they represent putative effectors that may be deployed to suppress host defences or bring about pathogen entry, but are targeted by the host and may therefore trigger cultivar-specific resistance in plants, or immune responses in human pathogens. This approach has proved very valuable in identifying effector genes in plantpathogenic oomycetes but has not yet been widely used in fungi (Win et al., 2006). A recent study has illustrated the power of association genetics and genome analysis to detect virulence-associated genes in the rice blast fungus Magnaporthe oryzae. Three novel effector-encoding genes were identified by screening for their presence/absence in a large collection of strains of the fungus, which showed a spectrum of resistance and susceptibility on diverse rice cultivars. The presence of each $M$. oryzae effector was associated with the presence of a cognate rice disease resistance gene, opening up the possibility of investigating the role of these effectors in plant defence suppression and how they are recognized by the products of these resistance genes (Yoshida et al., 2009). Other exciting applications of next-generation sequencing will include comparing the genomes of virulent and non-virulent isolates of the same species, comparing the genomes of closely related pathogenic and saprotrophic fungal species, and comparing geographically diverse pathogen collections. In this way it should prove possible to identify pathways and gene families necessary for virulence, host range and pathogen fitness.

High-throughput sequencing will, however, also provide a means of determining which genes are expressed during infection-related development and pathogenesis. Both Illumina and 454 sequencing can be adapted to analyse the transcriptome of any particular fungal species, providing information on both the identity and abundance of genes being transcribed under a defined set of conditions, and functional genomic information such as alternative splice sites. The relatively low cost and high accuracy of these platforms will ensure they are the procedures of choice for transcript analysis in the near future, and will eventually render microarray analysis obsolete. As the large-scale and inexpensive analysis of genomes and transcriptomes from many fungal species, under many different conditions, becomes routine, the functional genomic comparison of pathogenic and nonpathogenic fungi, leading to the identification of novel virulence determinants, will be achieved with unparalleled detail. In addition, massively parallel sequencing will be applied to the discovery of small RNAs (Ghildiyal \& Zamore, 2009) and the role they play in fungal physiology, chromatin immuno-precipitation studies and the elucidation of transcription regulatory networks in fungal development (Mitchell et al., 2009), and the role of epigenetic gene regulation in fungal development, physiology and virulence via genome-wide methylation analysis as recently demonstrated for plants (Corkus et al., 2008). 


\section{Analysis of the fungal proteome}

In systems biology, comparative genome analysis and functional genomics provide information on only a subset of the interactions that are occurring during growth and development in a living cell. In a hierarchy of interactions, the next level of complexity arises from the behaviour of the proteins that result from expression of each gene in the transcriptome. This layer of the interactome is being understood through the mathematical modelling of how proteins in the cell interact to form signalling pathways and networks. In turn, the data for such mathematical modelling are being generated by large-scale proteinprotein interaction studies which, while currently focused almost exclusively on the yeast interactome ( $\mathrm{Yu}$ et al., 2008) and other higher eukaryotes such as Caenorhabditis elegans (Li et al., 2004), will be applied to more fungi as the techniques involved become more widely adopted. Two general high-throughput approaches are currently used to study how proteins in the cell interact: determination of interacting protein partners following the purification from a cell extract of a known protein (the pull-down approach: Einarson, 2001); and yeast two-hybrid assays involving reporter gene expression (the binary approach: Fields \& Song, 1989).

Affinity-purification chromatography coupled to mass spectrometry enables proteins that are tagged with one or more affinity labels to be purified from cell extracts on a chromatography column specific for that label. Following elution from the affinity column, mass spectrometry is then used to identify the interacting partners of the protein that are co-eluted from the column. Similarly, proteins tagged with an antibody recognition epitope can be precipitated from a mixture of proteins in cell extracts using antibodies specific for the epitope tag. Interacting protein partners of the tagged protein will be co-precipitated and isolated from the cellular milieu for further analysis. This method is known as co-immunoprecipitation (Co-IP). Both methods have benefited from advances in the instrumentation used to analyse the purified protein complex. Peptides are first ionized by matrix-assisted laser desorption ionization (MALDI) and analysed on orthogonal time-of-flight (QTOF) instruments. The sensitivity of current MALDI-TOF systems ensures the accurate identification of interacting protein partners even if the purification method (affinity column or Co-IP) results in substantial loss of weakly interacting complexes. Furthermore, complexes containing more than two proteins can be identified in a physiological setting. This differs from the binary analysis of protein interactions, where (typically) two proteins are expressed in an artificial yeast host. In this assay, genes encoding two proteins of interest are expressed in a yeast host strain. One of the proteins (the bait) is fused to the DNA-binding domain of the yeast GAL4 protein, while the other (the prey) is fused to the activation domain of GAL4. If the two non-yeast bait and prey proteins physically interact, the DNA-binding and activation domains of GAL4 are brought into proximity at the GAL4 cognate receptor site, resulting in the transcription of a GAL4-dependent yeast reporter gene. While this assay is typically only used for binary analysis, and does not occur in physiologically relevant environments, it has the potential, unlike pull-down methods, to be massively scalable. Using vectors - such as the Gateway system - and yeast host strains of different mating types, libraries of yeast strains can be constructed that carry either all the genes of a particular fungus as bait, or all the genes of a particular fungus as prey. Mating the two libraries together and selecting for colony growth on medium that is not permissible to either host strain but is permissible when prey and bait interact will be a powerful tool for understanding the full range of protein-protein interactions in fungi.

A combination of both binary and pull-down approaches will provide unprecedented information on the interactions that occur between proteins in the cell. Making sense of those interactions requires bioinformatic resources and software developments that model the protein interaction data into experimentally testable cellular networks and protein signalling pathways. Such resources will lead to detailed models of fungal proteomes and how they change during different stages of development and in response to different environmental conditions and will reveal, for example, novel targets for drug development. In addition, information about how proteins interact with DNA (using chromatin immunoprecipitation, protein binding arrays and yeast one-hybrid assays) and cellular metabolites (using gas chromatography coupled to mass spectrometry) will be integrated with genomic, transcriptomic and proteomic data to provide a rich description of the dynamics of fungal cellular function at the molecular level.

\section{Analysis of the fungal metabolome}

Changes in transcription and, consequently, cellular protein abundance and composition inevitably result in metabolic changes within the cell. Biochemical pathways and the metabolites that flow through them produce specific chemical fingerprints representing the summation of cellular processes, including gene transcription levels and protein-protein interactions, at a given time and under given conditions. By extension, changes in the metabolic profile of a cell can be indicative of a cellular response to different biological stimuli. Advances in the quantification and identification of metabolites, and in the tools to interpret the resulting dataset, have led to the development of two similar fields of study devoted to relating chemical fingerprints to biological processes: metabonomics, which measures the global metabolic response of complex multicellular systems to different stimuli through time, and metabolomics, which aims to identify and quantify the total set of metabolites in a cell sample. The two terms are used interchangeably (Nicholson \& Lindon, 2008). Using NMR spectroscopy and GC-mass spectrometry for metabolite identification, these approaches provide an integrated view of the biochemistry of a cell. While these 
approaches have been used extensively in the medical field, for instance in the correlation of metabolites with disease states (for examples, see Nicholson \& Lindon, 2008), few uses of these technologies have yet been recorded in fungi. However, two recent publications demonstrate the potential of metabolomics for fungal research. In the rice blast fungus $M$. oryzae, chemical fingerprinting has demonstrated how metabolite profiles change during infection of host tissue (Parker et al., 2009). This work showed how malate and polyamines accumulated in plant cells rather than being used to generate defensive reactive oxygen species, and how the conversion of photoassimilate into mannitol and glycerol for carbon sequestration appeared to drive hyphal growth. Taken together, the results revealed how the fungus dynamically reprograms host metabolism during plant infection, both to suppress the host defence response to invasion and to direct plant metabolism for nutrient acquisition. In the soil saprophyte Aspergillus nidulans, recent chemical profiling of mutants of CclA, a Bre2 orthologue involved in histone $\mathrm{H} 3$ lysine-4 methylation, has demonstrated the production of novel secondary metabolites in these strains that are not synthesized by the wild-type (Bok et al., 2009). Inactivating CclA was shown to alter the chromatin landscape through disruption of lysine methylation and resulted in the expression of cryptic secondary metabolite clusters, genes encoding the enzymes for secondary metabolite production that are usually silent. In consequence, HPLC profiles and NMR analysis identified six new secondary metabolites produced by the CclA loss-of-function mutant strains, including Emodin, a compound not previously detected in A. nidulans and known to have many beneficial properties including antimutagenic, anti-tumour and immunosuppressive activities. In the future, such metabolite analysis coupled to genetic manipulations will be essential tools in the discovery of new and beneficial natural products.

\section{Phenotype microarray development for high- throughput gene functional analysis of fungi}

One strength of fungal research lies in the ease with which some fungi can be genetically manipulated. Targeted approaches, using forward and reverse genetic selections, have led to a deep understanding of both the structural genes that underpin cellular processes, and the regulators that control their expression. How will a systems biology approach to fungal research drive future developments in our understanding of the regulatory networks that control gene expression in response to changes in, for example, ambient $\mathrm{pH}$, nitrogen source utilization and carbon availability? Such changes, and mutations in the genes that regulate gene expression in response to these changes (for example $p a c C$, areA and creA), result in altered gene expression patterns, protein-protein interactions and metabolite production which can be measured by the platforms described above. In contrast, phenotypic analysis reveals the effect of the genetic mutation or growth condition change at the physiological level. The devel- opment of high-throughput phenotype microarrays for rapidly assessing the effects of mutations in regulatory genes on fungal physiology is therefore an attractive prospect for fungal research and will reveal the summation of gene expression, protein interaction and biochemical changes that accompany genetic and physiological perturbations of fungi. Tanzer et al. (2003) overcame the difficulties of using filamentous fungi compared to unicellular organisms in phenotypic microarrays. They used high-throughput microtitre-plate-based nutrient utilization assays in the following diverse applications: to perform global analyses of nitrogen source utilization in $A$. nidulans wild-type and a series of strains containing mutations in the areA gene; to compare nutrient utilization in two fungal species in the presence of the glutamine synthetase inhibitor glufosinate; and to compare carbon catabolite repression characteristics in four filamentous fungal species, including a human pathogen and two plant pathogens, based on resistance to allyl alcohol. These experiments demonstrated the utility of high-throughput phenotype microarray analysis, chemical characterization and gene function determination in filamentous fungi, and we can expect such approaches to be used to determine the physiological effect of other regulatory mutations, such as those affecting $p a c C$ and $c r e A$ homologues. In addition, phenotype microarrays can be used to characterize mutations in genes that integrate multiple regulatory pathways. The TPS1 gene of M. oryzae, encoding trehalose-6-phosphate synthase, is one such gene, which is required for integrating carbon and nitrogen metabolism through the control of NADPH levels in response to glucose 6-phosphate (Wilson et al., 2007). This integration is crucial for the fungus to cause rice blast disease, and a full phenotypic characterization of TPS1 will be needed to understand how this fungus causes disease.

\section{High-throughput techniques for large-scale functional genomic analyses of fungi}

Systems-level approaches to understanding the complex interactions that underlie cellular function will require experimental validation of the role of signalling pathways and effectors in fungal physiology, and the characterization of fungal genes likely to be involved in undesirable functions such as those related to human or plant pathogenesis. The wealth of data generated by the approaches described above necessitates the development of high-throughput gene disruption protocols and other strategies to rapidly delineate the function of the vast number of genes implicated in the cellular networks. In fungi, traditional methods of gene functional analysis involving single gene replacement, protein localization studies and phenotype screening are already being replaced by high-throughput methodologies. For example, rapid targeted gene replacement of single genes can now utilize double-jointed PCR combined with recipient strains lacking components of the non-homologous DNA endjoining pathway, Ku70 and Ku80 (Kershaw \& Talbot, 2009; 
Villalba et al., 2008), thereby simultaneously eliminating time-consuming disruption vector construction - with its associated and cumbersome cloning and ligation steps and the need to screen large numbers of transformants to identify progeny carrying homologous replacements at the loci of interest. Beyond single-gene analysis, RNA interference-mediated gene silencing technologies are being developed for the rapid functional analysis of many genes: for instance the silencing of all genes involved in a particular pathway, or the silencing of all members of a multigene family (Nguyen et al., 2008). In other developments, the traditional two-step forward genetics screen for identifying novel gene functions - whereby mutations in unknown genes are generated by mutagenesis followed by selection, and the unknown gene is identified by complementation with a genomic library - is being replaced by single-step gene tagging analyses. Using either agrobacterium- or transposon-mediated transformation of fungal strains (Tucker \& Orbach, 2007; Brown \& Holden, 1998), gene disruptions can be first selected following the classical method, and then immediately identified using the genetic tag introduced by these methods.

For protein localization studies, Gateway vectors (Zhu et al., 2009) can be used to tag proteins with fluorescently labelled markers such as GFP, or alternatively, GFP fusion proteins can be obtained directly by overlapping PCR using PCR products of the genes of interest combined with the GFP PCR product. In addition, advances in live-cell imaging can localize the GFP-tagged proteins with unprecedented precision.

Finally, high-throughput phenotypic analysis of mutants generated in the large-scale gene disruption studies will be necessary. These will include comprehensive nutritional profiling of mutant strains in 96- or 384-well formats, as discussed above, and the large-scale analysis of perturbations to biochemical pathways through the measurement, in 96well format, of enzyme activities in these mutant strains.

Taken together, we can expect systems biology coupled with high-throughput gene disruption strategies to result in great advances in the identification and characterization of the fundamental cellular processes and networks that govern fungal physiology. This will enable us to better enhance desirable fungal traits - such as the production of beneficial secondary metabolites to combat disease - while developing strategies to mitigate the undesirable fungal attributes related to human and plant disease that are currently impacting human societies.

\section{References}

Bok, J. W., Chiang, Y.-M., Szewczyk, E., Reyes-Domingez, Y., Ashley, D., Davidson, A. D., Sanchez, J. F., Lo, H.-C., Watanabe, K. \& other authors (2009). Chromatin-level regulation of biosynthetic gene clusters. Nat Chem Biol 5, 462-464.

Brown, J. S. \& Holden, D. W. (1998). Insertional mutagenesis of pathogenic fungi. Curr Opin Microbiol 1, 390-394.
Calvo, A. M., Wilson, R. A., Bok, J. W. \& Keller, N. P. (2002). Relationship between secondary metabolism and fungal development. Microbiol Mol Biol Rev 66, 447-459.

Corkus, S. J., Feng, S., Zhang, X., Chen, Z., Merriman, B., Haudenschild, C. D., Pradhan, S., Nelson, S. F., Pellegrini, M. \& Jacobsen, S. E. (2008). Shotgun bisulphite sequencing of the Arabidopsis genome reveals DNA methylation patterning. Nature 452, 215-219.

Cornell, M. J., Alam, I., Soanes, D. M., Wong, H. M., Hedeler, C., Paton, N. W., Rattray, M., Hubbard, S. J., Talbot, N. J. \& Oliver, S. G. (2007). Comparative genome analysis across a kingdom of eukaryotic organisms: specialization and diversification in the fungi. Genome Res 17, 1809-1822.

Desjardins, A. E. (2003). Gibberella from A (venaceae) to Z (eae). Annu Rev Phytopathol 41, 177-198.

Einarson, M. B. (2001). Detection of protein-protein interactions using the GST fusion protein pulldown technique. In Molecular Cloning: a Laboratory Manual, 3rd edn, pp. 18.55-18.59. Edited by J. Sambrook \& D. W. Russell. Cold Spring Harbor, NY: Cold Spring Harbor Laboratory.

Fields, S. \& Song, O. (1989). A novel genetic system to detect protein-protein interactions. Nature 340, 245-246.

Galán, J. E. \& Wolf-Watz, H. (2006). Protein delivery into eukaryotic cells by type III secretion machines. Nature 444, 567-573.

Ghildiyal, M. \& Zamore, P. D. (2009). Small silencing RNAs: an expanding universe. Nat Rev Genet 10, 94-108.

Harris, S. D., Turner, G., Meyer, V., Espeso, E. A., Specht, T., Takeshita, N. \& Helmstedt, K. (2009). Morphology and development in Aspergillus nidulans: a complex puzzle. Fungal Genet Biol 46, S82S92.

Hedayati, M. T., Pasqualotto, A. C., Warn, P. A., Bowyer, P. \& Denning, D. W. (2007). Aspergillus flavus: human pathogen, allergen and mycotoxin producer. Microbiology 153, 1677-1692.

Kershaw, M. J. \& Talbot, N. J. (2009). Genome-wide functional analysis reveals that infection-associated fungal autophagy is necessary for rice blast disease. Proc Natl Acad Sci U S A 106, 15967-15972.

Li, S., Armstrong, C. M., Bertin, N., Ge, H., Milstein, S., Boxem, M., Vidalain, P. O., Han, J. D., Chesneau, A. \& other authors (2004). A map of the interactome network of the metazoan C. elegans. Science 303, 540-543.

Mitchell, T. K., Dean, R. A., Xu, J.-R., Zhu, H., Oh, Y. Y. \& Rho, H.-S. (2009). Protein chips and chromatin immunoprecipitation emerging technologies to study macromolecule interactions in $M$. grisea. In Advances in Genetics, Genomics and Control of Rice Blast Disease, pp. 73-82. Edited by G. L. Wang \& B. Valent. Dordrecht: Springer.

Nguyen, Q. B., Kadotani, N., Kasahara, S., Tosa, Y., Mayama, S. \& Nakayashiki, H. (2008). Systematic analysis of calcium signalling proteins in the genome of the rice blast fungus Magnaporthe oryzae using a high-throughput RNA silencing system. Mol Microbiol 68, 1348-1365.

Nicholson, J. K. \& Lindon, J. C. (2008). Metabonomics. Nature 455, 1054-1056.

Parker, D., Beckmann, M., Zubair, H., Enot, D. P., Caracuel-Rios, Z., Overy, D. P., Snowdon, S. J., Talbot, N. J. \& Draper, J. (2009). Metabolomic analysis reveals a common pattern of metabolic reprogramming during invasion of three host plant species by Magnaporthe grisea. Plant J 59, 723-737.

Rispail, N., Soanes, D. M., Ant, C., Czajkowski, R., Grünler, A., Huguet, R., Perez-Nadales, E., Poli, A., Sartorel, E. \& other authors (2009). Comparative genomics of MAP kinase and calcium- 
calcineurin signalling components in plant and human pathogenic fungi. Fungal Genet Biol 46, 287-298.

Soanes, D. M., Richards, T. A. \& Talbot, N. J. (2007). Insights from sequencing fungal and oomycete genomes: what can we learn about plant disease and the evolution of pathogenicity? Plant Cell 19, 33183326.

Staples, R. C. (2000). Research on the rust fungi during the twentieth century. Annu Rev Phytopathol 38, 49-69.

Tanzer, M. M., Arst, H. N., Skalchunes, A. R., Coffin, M., Darveaux, B. A., Heiniger, R. W. \& Shuster, J. R. (2003). Global nutritional profiling for mutant and chemical mode-of-action analysis in filamentous fungi. Funct Integr Genomics 3, 160-170.

Tucker, S. L. \& Orbach, M. J. (2007). Agrobacterium-mediated transformation to create an insertion library in Magnaporthe grisea. In Plant-Pathogen Interactions, pp. 57-68. Edited by P. C. Ronald. Totowa, NJ: Humana Press.

Villalba, F., Collemare, J., Landraud, P., Lambou, K., Brozek, V., Cirer, B., Morin, D., Bruel, C., Beffa, R. \& Lebrun, M.-H. (2008). Improved gene targeting in Magnaporthe grisea by inactivation of MgKU80 required for non-homologous end joining. Fungal Genet Biol 45, 68-75.

Whisson, S. C., Boevink, P. C., Moleleki, L., Avrova, A. O., Morales, J. G., Gilroy, E. M., Armstrong, M. R., Grouffaud, S., van West, P. \& other authors (2007). A translocation signal for delivery of oomycete effector proteins into host plant cells. Nature 450, 115-118.
Wilson, R. A. \& Talbot, N. J. (2009). Under pressure: investigating the biology of plant infection by Magnaporthe oryzae. Nat Rev Microbiol 7, 185-195.

Wilson, R. A., Jenkinson, J. M., Wang, Z.-Y., Gibson, R. P., Littlechild, J. A. \& Talbot, N. J. (2007). Tps1 regulates the pentose phosphate pathway, nitrogen metabolism and fungal virulence. $E M B O ~ J ~ 26,3673-3685$.

Win, J., Kanneganti, T. D., Torto-Alalibo, T. \& Kamoun, S. (2006). Computational and comparative analyses of 150 full-length cDNA sequences from the oomycete plant pathogen Phytophthora infestans. Fungal Genet Biol 43, 20-33.

Yadav, G., Gokhale, R. S. \& Mohanty, D. (2009). Towards prediction of metabolic products of polyketide synthases: an in silico analysis. PLOS Comput Biol 5, e1000351.

Yoshida, K., Saitoh, H., Fujisawa, S., Kanzaki, H., Matsumura, H., Yoshida, K., Tosa, Y., Chuma, I., Takano, Y. \& other authors (2009). Association genetics reveals three novel avirulence genes from the rice blast fungal pathogen Magnaporthe oryzae. Plant Cell 21, 1573-1591.

Yu, H., Braun, P., Yildirim, M. A., Lemmens, I., Venkatesan, K., Sahalie, J., Hirozane-Kishikawa, T., Gebreab, F., Li, N. \& other authors (2008). High-quality binary protein interaction map of the yeast interactome network. Science 322, 104-110.

Zeigler, R. S., Leong, S. A. \& Teng, P. S. (1994). Rice Blast Disease. Wallingford, UK: CAB International.

Zhu, T., Wang, W., Yang, X., Wang, K. \& Cui, Z. (2009). Construction of two Gateway vectors for gene expression in fungi. Plasmid 62, 128-133. 